\title{
High Dynamic Range Images from Digital Cameras Raw Data
}

\author{
Géraldine Joffre* \\ MultimediaPhoto
}

\author{
William Puech ${ }^{\dagger}$ \\ LIRMM-CNRS Montpellier
}

\author{
Frédéric Comby \\ LIRMM-CNRS Montpellier
}

\author{
Jacques Joffre $^{\S}$ \\ ENSCM Montpellier
}

Several methods have been proposed to reconstruct a High Dynamic Range (HDR) image from differently exposed photographs that are either scanned films or firmware-processed outputs of digital cameras [Debevec et al, 1997]. We use instead the unprocessed sensor data of digital cameras and show how this simplifies the HDR image reconstruction and more accurately reproduces color information from the original scene.

\section{Linearity check}

Though sensors of standard digital cameras can hold up to 12 bits of digitized signal, in-camera processes scale it down to just 8 bits of tonal information. Using raw output from the camera sensors provides a larger coverage of the dynamic range at a higher precision. It also enables a more accurate computation of the luminance value since the response of the sensors is considered to be linear relative to the input signal.

To check this important property of linearity, we photographed an HDR scene under 36 exposures spaced by $1 / 3$ EV using a Canon 1D Mark II camera set to output raw data. We then plotted the raw data pixel values relative to exposure for four levels of luminance (figure 1).

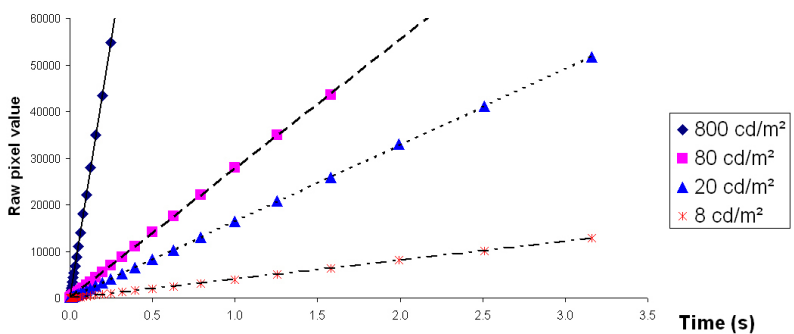

Figure 1: Canon 1D Mark II raw data tonal response.

Having linear data at our disposal eliminates the need for calibration. It also makes the HDR image calculation depend on hardware specification only and not on firmware processes that may differ across the exposure range and vary depending on the characteristics of the scene.

\section{Color gamut}

Algorithms for constructing HDR images focus on the camera tonal rather than color response. An exception is the calibration technique in [Goesele et al 2001] where ICC profiles are used. We take the same approach, except that we profile the camera sensor instead of the low dynamic range output resulting from in-camera processing. This has for advantage to preserve the full color gamut of the

\footnotetext{
*e-mail: gjoffre@multimediaphoto.com

†e-mail:william.puech@lirmm.fr

†e-mail:comby@lirmm.fr

§e-mail:jacques.joffre@enscm.fr
}

capturing device, contrary to processed data converted to the lower gamut of standard display devices. We characterized three different camera models by photographing an IT8 color chart and determining the $3 \times 3$ matrix relating the camera raw RGB values to device independent CIE-XYZ coordinates. We then calculated the volume of the convex hull of the Lab values obtained by converting 500 points of a test image with each matrix and did the same with the sRGB color space for comparison (Table 1).

\begin{tabular}{|l|r|r|}
\hline & Volume & Ratio to sRGB \\
\hline sRGB & 560,958 & $1: 1$ \\
\hline Canon D30 & $1,196,562$ & $2.13: 1$ \\
\hline Canon 1D Mark II & $1,406,893$ & $2.51: 1$ \\
\hline Fuji S3 Pro & $1,428,578$ & $2.55: 1$ \\
\hline
\end{tabular}

Table 1: Gamut volume comparison.

Standard HDR image formats do not impose any constraint on the size of the gamut that can be properly quantized. Our results show that HDR images constructed from characterized unprocessed data can represent at least twice as much visible colors than when constructed from processed outputs with sRGB as color space.

We then compared the effect of tone mapping two HDR images created from the same captures, one from unprocessed and the other from processed data. Both images were tone mapped using the technique in [Drago et al. 2003] and selecting the proper RGB to $\mathrm{XYZ}$ conversion. As expected, tone mapping the unprocessed image results in a higher number of color values that lie outside the gamut of standard display devices.

\section{Conclusions and future directions}

We showed that HDR reconstruction using unprocessed instead of processed outputs of digital cameras eliminates the tonal calibration step and preserves more color information of the original scene. We are now evaluating the impact of an extended color gamut for HDR images. Our future work will focus on selecting an appropriate gamut mapping technique for HDR images and incorporating it into the above cited tone mapping algorithm.

\section{References}

Debevec, P., AND MaliK, J. 1997. Recovering high dynamic range radiance maps from photographs. In Proceedings of SIGGRAPH 1997, Annual Conference Series, ACM, 369-378.

Drago, F., Myszkowski, K., Annen, T., And Chiba, N. 2003. Adaptive logarithmic mapping for displaying high contrast scenes. In Proceedings of Eurographics 2003, The Journal of Computer Graphics Forum, 419-426.

Goesele, M., Heidrich, W., ANd Seidel, H. 2001. Color calibrated high dynamic range imaging with icc profiles. In Proceedings of the Ninth Color Imaging Conference, 286-290. 